Ann. Génét. Sél. anim., I972, 4 (3), 423-444.

\title{
VARIABILITÉ GÉNÉTIQUE DE LA CROISSANCE AVANT SEVRAGE DANS LES RACES CHAROLAISE ET LIMOUSINE
}

\author{
H. A. MOLINUEVO $\left({ }^{1}\right)$ et B. VISSAC \\ Station de Génétique quantitative et appliquée, \\ Centre national de Recherches zootechniques, I. N. R. A., \\ 78 - Jouy-en-Josas
}

\section{RÉSUMÉ}

On a pu constituer, à partir du fichier de contrôle de croissance des veaux de races à viande, I2 36I couples mère-produit en charolais et 2928 en limousin, couples pour lesquels on disposait simultanément des poids à la naissance, 90 , I 20 et $\mathrm{I} 80$ jours. Les coefficients d'héritabilité et de corrélation génétique entre ces critères ont été estimés à partir des coefficients de régression mèreproduit intra-élevage, année et classe d'âge des mères.

Les coefficients d'héritabilité du poids qui ont, en général, une valeur maximum à la naissance $(0,15-0,30)$, passent par un minimum vers $3-4$ mois $(0,00-0,17)$ et augmentent de 4 à 6 mois $(0,10-0,24)$.

Les valeurs de ces coefficients sont en général supérieures pour les veaux mâles par rapport aux femelles; on a pu vérifier que cette différence que l'on pourrait interpréter en invoquant l'incidence de gènes liés au sexe était en grande partie la conséquence des écarts entre sexes dans la variance phénotypique des perfornıances.

Ces coefficients, ainsi que la variabilité génétique, exprimée en valeur absolue ou relative (coefficient de variation génétique) sont en général supérieurs en race charolaise pour des poids homologues.

Quant aux coefficients de corrélation génétique, leurs valeurs sont, dans l'ensemble, plus élevées en race limousine.

Ces résultats sont, en général, conformes à ceux obtenus dans d'autres races, anglosaxonnes en particulier. Leur analyse permet de prévoir dans le cas des veaux mâles, qu'il sera plus facile, en race charolaise de sélectionner la croissance post-natale indépendamment du poids à la naissance.

\section{INTRODUCTION}

Les races à viande françaises connaissent depuis une dizaine d'années une expansion considérable dans les pays d'élevage extensif de bovins à viande. Elles sont, dans un premier temps, croisées avec les races anglo-saxonnes ou les zébus exploités

(1) Adresse actuelle: I.N.T.A., Balcarce, Pcia Buenos-Aires - Argentine. 
jusqu'ici dans ces pays. Il ressort des expérimentations réalisées dans la plupart des pays que si l'emploi de ces races - de la charolaise notamment (VISSAC, I970) entraîne une nette augmentation du potentiel de croissance musculaire, il s'accompagne également d'un accroissement des difficultés de vêlage par incompatibilité morphologique fœto-maternelle (BELIC et MENISSIER, I968). Cette incompatibilité est liée, d'une part à un effet direct favorable sur les dimensions du veau à la naissance, d'autre part à un effet maternel de réduction de l'ouverture pelvienne (RÉGIS, I969). On est ainsi conduit à étudier, entre autres, les possibilités de sélection de la croissance post-natale de ces races indépendamment du poids à la naissance. Cette croissance, exprimée le plus souvent par le poids ou la conformation au sevrage, était seule considérée jusqu'ici dans la sélection des races bovines à viande. Une telle étude nécessite de connaître les paramètres génétiques de la croissance pondérale pré- et post-natale des veaux dans les races charolaise et limousine. Si ces paramètres ont déjà été estimés à partir des résultats du contrôle de descendance des taureaux de ces races en croisement avec des femelles de races diverses pour la production de veaux de boucherie (Poujardieu et Vissac, I968), ils correspondent en fait à une situation très particulière, tant en ce qui concerne la variabilité génétique analysée (aptitude au croisement), que vis-à-vis du type de production concerné.

L'objet du présent mémoire est d'obtenir des estimations en races pures à partir des données du contrôle de croissance effectué depuis ro à I 5 ans dans les zones françaises de sélection des races charolaise et limousine où les animaux sont élevés dans des conditions assez comparables à celles en usage dans la plupart des pays d'élevage à viande.

\section{I. - MATÉRIEL ANIMAL}

On a utilisé les données du contrôle de croissance effectué dans les élevages chavolais et limousins pendant la période comprise entre les années 1958 et 1969, incluses.

Nous avons sélectionné tous les couples mère-produit dont on connaissait ou dont on avait pu calculer les poids à la naissance, à 90 jours, à I 20 jours, et au sevrage (I 80 jours), pour chaque membre de la paire. On a pu disposer ainsi de :

- I 2 36I couples en race charolaise, dont 5327 correspondent à des produits mâles et, 7034 à des produits femelles;

- 2928 couples en race limousine, dont I 499 avec des produits mâles et I 429 avec des produits femelles.

Les animaux charolais proviennent principalement des départements du Cher, de la Nièvre, de la Saône-et-Loire, de l'Allier et de la Creuse. Les limousins sont concentrés surtout dans les départements de la Haute-Vienne, de la Corrèze et de la Creuse.

Nous préciserons successivement les conditions d'élevage des veaux pendant la phase de croissance considérée dans cette étude, les contrôles qui sont réalisés et leur utilisation en vue d'exprimer la croissance des veaux, la structure génétique enfin de notre échantillon telle qu'elle résulte des systèmes d'accouplement pratiqués par les éleveurs.

\section{I. x. - Méthode d'élevage}

Il convient de distinguer les conditions d'élevage utilisées en race charolaise de celles pratiquées pour la race limousine.

Dans le premier cas, on est en présence d'un système extensif classique. Les naissances sont échelonnées du mois de janvier aux mois d'avril-mai, les femelles étant mises à la reproduction à partir du mois de mars pour les génisses, et du mois d'avril (mise à l'herbe) pour les vaches. 
Les veaux nés en janvier, février et mars à l'étable restent en stabulätion avec leurs mères jusqu'à la mise en herbe. Les naissances postérieures à cette époque ont lieu en général en stabulation, les veaux sortant avec leurs mères dès l'âge de I 5 jours.

Le troupeau de mères allaitantes et de veaux est exploité au pâturage, en général sans complémentation, jusqu'au mois de juillet. A partir de ce mois, et selon les conditions climatiques, une complémentation peut être fournie aux veaux dans certains troupeaux; cette complémentation est réservée aux veaux mâles dans la plupart des cas. Ce traitement différentiel est particulièrement important à considérer à partir du mois de septembre dans les élevages orientés vers la production de taurillons reproducteurs. A partir de cette date, on peut également considérer que, dans ces élevages, les taurillons choisis pour la reproduction sont soumis à un régime plus intensif que les autres taurillons. En fait, notre étude ne portant que sur les poids de la naissance jusqu'à 6 mois, on peut penser que ce dernier facteur n'a eu qu'une incidence faible.

En race limousime, il faut distinguer trois systèmes d'élevage caractéristiques :

\section{II. Système à l'auge.}

Il s'agit du système traditionnellement pratiqué dans le passé par les éleveurs limousins. Les veaux vivent en stabulation toute l'année, tandis que les mères sont en stabulation en hiver et sortent au pâturage en été. Elles rentrent deux fois par jour à l'étable pour allaiter leurs veaux qui reçoivent une alimentation concentrée supplémentaire à partir de l'âge de 3 mois. Les éleveurs pratiquent l'insémination artificielle plus fréquemment que dans les systèmes suivants.

\section{I2. Système de plein-air intégral.}

Les animaux qui appartiennent en moyenne à des troupeaux de grands effectifs vivent toute l'année dehors, le plus souvent au pâturage. Le foin peut être distribué en complément l'hiver. La saillie est pratiquée en monte libre de telle façon que les naissances soient groupées au printemps, principalement de février à avril. Les veaux restent avec les mères jusqu'au sevrage. Ils ont en général accès, dès le deuxième mois, à un supplément concentré. Ce système est donc équivalent à celui utilisé en Charolais.

\section{13. Système de semi-plein-air.}

Il existe en fait de nombreux systèmes intermédiaires entre le système traditionnel (dit à l'auge) et le système extensif (dit de plein-air intégral). Souvent les animaux rentrent à l'étable en hiver où se produisent les naissances. La conduite pendant l'été peut se distinguer de celle du système de plein-air en ce sens que les veaux qui restent au pâturage le jour rentrent parfois en stabulation la nuit.

Il était difficile de distinguer ces 3 systèmes dans notre étude, car si des élevages se classent facilement dans chacune de ces 3 catégories, un élevage donné a pu connaître, au cours de la période considérée, plusieurs stades de l'évolution ci-dessus.

\section{2. - Contrôle de la croissance}

Dans tous les cas, le poids du veau à la naissance est fourni par l'éleveur qui pèse son veau dans les $48 \mathrm{~h}$ qui suivent la mise bas, à l'aide d'une bascule ou d'une balance romaine. Les veaux sont ensuite pesés à des périodes différentes suivant le système considéré :

- En système d'élevage de plein-air (race chavolaise et partie de la race limousine) on pèse avant la mise à l'herbe (fin mars et début avril), avant la période de sécheresse estivale et le début de la complémentation des veaux (fin juin, début juillet), au sevrage enfin (de fin septembre à milieu novembre).

- En système d'élevage à l'auge (race limousine) où les vêlages sont répartis sur toute l'année, une pesée trimestrielle est réalisée pour l'ensemble des veaux de l'élevage.

Les poids à 90 , r 20 et I 80 jours sont calculés à partir des résultats de ces pesées par interpolation linéaire entre les 2 pesées qui encadrent les âges de références ci-dessus. Les deux premiers âges correspondent à la période pour laquelle la corrélation entre le poids de l'animal et la quantité de lait maternel consommé est maximum. Ce sont, par ailleurs, (Pou JARdiEU, I969) les âges les plus voisins de celui auquel est pratiquée la pesée correspondant au début de la période de sécheresse (juin, juillet).

En ce qui concerne l'âge de référence, nous avons retenu r80 jours, de préférence à 2 ro jours qui est utilisé classiquement dans les travaux américains sur bovins à viande, en vue d'exprimer le poids au sevrage, car l'engraissement et la vente précoce de certains veaux mâles destinés à 
la reproduction risquait de réduire l'effectif de notre échantillon et d'introduire un biais. On note déjà une disproportion importante entre les effectifs de veaux mâles et femelles de race charolaise.

Nous remarquons pour la suite de ce travail que, étant donné la fréquence des pesées, les poids adjacents (90 et $\mathbf{I} 20$ ou $\mathbf{1} 20$ et I 80 ) peuvent présenter entre eux une liaison authentique du fait qu'ils ont été calculés parfois à partir d'une observation, voire peut-être de 2 observations communes. Ceci est particulièrement vrai pour les poids à 90 et 120 jours.

\section{3. - Structuve génétique de l'échantillon}

Notre échantillon comportait, en race charolaise, une majorité de femelles soumises à la monte naturelle. En race limousine, par contre, l'insémination artificielle était le mode de reproduction le plus répandu : bien que ces échantillons ne soient pas rigoureusement représentatifs des populations de femelles de ces 2 races, on signalera (statistique agricole, I970) que le taux global de vaches inséminées est de 32, I p. Ioo en race charolaise contre 77,6 p. Ioo en race limousine. Dans le cas de la monte naturelle, les taureaux ne sont utilisés en général que dans un seul troupeau sur un effectif de 20 à 70 vaches, les génisses étant souvent accouplées avec un géniteur différent de celui utilisé pour les vaches du même troupeau et choisi en raison de son format plus réduit et de son jeune âge. Un taureau (tabl. I) est en général utilisé de I à 3 ans suivant les cas (VISSAC, I970). Dans le cas de l'insémination artificielle, on peut considérer que les accouplements sont réalisés au hasard à partir des taureaux de service des centres, taureaux ayant pour la plupart subi favorablement les épreuves de contrôle de descendance sur la croissance et la conformation de leurs veaux ; les éleveurs concernés ici, et qui sont orientés vers la sélection, ne participent pas, en général, aux épreuves de la descendance des jeunes taurillons achetés par les centres.

\section{TABLEAU I}

Age moyen des taureaux (en années) par catégorie d'âge des mères

\begin{tabular}{l|c|c|c}
\hline \hline \multirow{2}{*}{ Race } & \multicolumn{3}{|c}{ No de vêlage des mères } \\
\hline limousine & 1 & 2,3 et '́t & 5 et + \\
\hline charolaise & 3,02 & 3,69 & 3,95 \\
\hline \hline
\end{tabular}

\section{II. — MÉTHODE S'TATISTIQUE}

\section{I. - Modèle d'analyse}

En raison de la structure génétique particulière de notre échantillon où domine la monte naturelle et où sont confondus partiellement (voir ci-dessus) les effets du troupeau, de l'année, de l'âge des femelles et du taureau (père des veaux), il n'était pas possible d'envisager une estimation des paramètres génétiques par comparaison des performances de $\mathrm{I} / 2$ frères (ou sœurs) paternels; nous avons donc retenu comme seule méthode d'analyse de la variabilité génétique, les comparaisons mère-enfant. Le nombre réduit de taureaux utilisés ainsi que la sélection importante effectuée pour choisir ces taureaux excluaient, par ailleurs, l'étude des relations pèrerenfant. Ces comparaisons ont été effectuées séparément pour les veaux de même race et de même sexe.

Les couples analysés ont été classés suivant la hiérarchie :

- année et no de vêlage de la mère ;

— élevage où étaient élevés à la fois la mère et le produit. 
Le premier niveau de cette classification englobe les effets moyens sur la croissance des veaux, de l'année, de l'âge des mères et partiellement du taureau dans la mesure où il y a monte naturelle et où un taureau déterminé est réservé aux génisses. En ce qui concerne la croissance des mères, le premier niveau de classification englobe en partie l'effet de l'année, dans la mesure où les femelles ont vêlé pour la première fois au même âge ( 3 ans) et ont eu ensuite un veau tous les ans ; ces conditions sont en général remplies, les génisses vêlant dans 95 p. Ioo des cas à 3 ans, et les femelles, infertiles une année donnée, étant éliminées de la reproduction.

Le deuxième niveau de classification correspond aux différences de croissance des veaux entre élevages pour une même année et des mères de même âge.

Pour les mères: Le modèle théorique d'analyse de la variance s'écrit alors :

$$
\mathrm{X}_{i j k l}=\mu_{i}+\mathrm{A}_{i j}+\mathrm{E}_{i j k}+x_{i j k l}
$$

nù

$\mathrm{X}_{i j k l}=$ valeur du caractère $i$ chez la mère contrôlée pour la I me paire correspondant à la $j$ me catégorie année-numéro de vêlage, dans le $k$ me élevage.

$\mu_{i}=$ moyenne commune à toutes les observations du caractère $i$.

$\mathrm{A}_{i j}, \mathrm{E}_{i j k}=$ variables aléatoires représentant un effet particulier commun respectivement à la $j$ me catégorie-année-no de vêlage et au $k$ me élevage; elles ont des espérances nulles et des variances $\sigma_{A i}^{2}, \sigma_{\mathbf{E}}^{2}$

$x_{i j k l}=$ variable aléatoire représentant la déviation relative à la $l$ me mère dans le $k$ me élevage et dans la $j$ me catégorie année-no de vêlage ; $x_{i j k l}$ a une espérance nulle et une variance $\sigma_{x}^{2}$.

Pour les produits :

où

$$
\mathrm{Y}_{i j k l}=\mu_{i}^{\prime}+\mathrm{A}_{i j}^{\prime}+\mathrm{E}_{i j k}^{\prime}+y_{i j k l}
$$

$\mathrm{Y}_{i j k l}, \mu_{i}^{\prime}, \mathrm{A}_{i j}^{\prime}, \mathrm{E}_{i j k}^{\prime}, y_{i j k l}$ ont la même signification que précédemment.

$\mathrm{A}_{i j}^{\prime}, \mathrm{E}_{i j k}^{\prime}, y_{i j k l}$ ont des espérances nulles et des variances respectivement égales à

$$
\sigma_{\mathbb{A}_{i}}^{2}, \sigma_{\mathbb{E}_{i}}^{2}, \sigma_{\underline{y}_{i}}^{2}
$$

\section{2. - Estimation des paramètres génétiques}

Pour chaque caractère, aussi bien chez la mère que chez le produit, nous avons effectué une décomposition de la variance suivant un schéma hiérarchique, et obtenu des estimations des variances ci-dessus :

$$
\sigma_{A i}^{2}, \sigma_{A c^{\prime}}^{2}, \sigma_{\mathrm{E}_{i}}^{2}, \sigma_{\mathrm{E}_{i}^{\prime}}^{2}, \sigma_{x_{i}}^{2}, \sigma_{y_{i}}^{2}
$$

Une analyse de covariance a été réalisée suivant le même modèle pour chaque paire $\left(\mathrm{X}_{i}, \mathrm{Y}_{i}\right)$ à l'intérieur de chaque échantillon de veaux de même race et de même sexe. On a de même obtenu les estimations des covariances :

$$
\sigma_{x_{i} y_{i}}, \sigma_{\mathbf{E}_{l} \mathbf{E}^{\prime}{ }_{i}}, \sigma_{\mathrm{A}_{i} \mathbf{A}^{\prime}{ }_{i}}
$$

La covariance résiduelle $\left(\sigma_{x_{i} y i}\right)$ entre les caractères $i$ mesurés chez les mères et chez les produits estime la moitié de la variance additive du caractère $i$ :

$$
\sigma_{x_{i} y_{i}}=\frac{\mathrm{I}}{2} \sigma_{g_{i}}^{2}
$$

Sur ces bases, l'héritabilité $\left(h_{i}^{2}\right)$ a été estimée par la régression mère-produit (LusH, I940):

$$
h^{2}=2 b=\frac{2 \sigma_{x_{i} y_{i}}}{\sigma_{x_{i}}^{2}}=\frac{\sigma_{i \cdot i}^{2}}{\sigma_{x_{i}}^{2}}
$$

L'écart-type de l'héritabilité a été calculé à l'aide de la formule :

$$
s_{h_{i}}{ }^{2}=2 s_{b}=2 \sqrt{\frac{\Sigma_{\mathrm{Y}^{2}}-\left(\Sigma_{\mathrm{XY}}\right)^{2} / \Sigma_{\mathrm{X}^{2}}}{\Sigma_{\mathrm{X}^{2}}}}
$$

où $\Sigma_{X^{2}}, \Sigma_{Y^{2}}, \Sigma_{X Y}$ sont les sommes des carrés et coproduits relatifs aux variables centrées résiduelles. 
De même, la covariance résiduelle $\left(\sigma_{x_{i} y_{i}}, i \neq i^{\prime}\right)$ estimée suivant le modèle hiérarchique ci-dessus entre le caractère $i$ mesuré chez les mères et le caractère $i^{\prime}$ mesuré chez les produits, estime la moitié de la covariance génétique entre les caractère $i$ et $i^{\prime}$ :

$$
\text { pour } i \neq i^{\prime}, \sigma_{x i y i^{\prime}}=\frac{\mathbf{I}}{2} \sigma_{g_{\imath} g_{i^{\prime}}}
$$

Les corrélations génétiques ont été estimées d’après la méthode proposée par HAzEL (I943) :

$$
v_{g_{i} g_{i}^{\prime}}=\frac{\sigma_{x_{i} y_{i}^{\prime}}+\sigma_{x_{i^{\prime} y_{i}}}}{\sigma_{x_{i} y_{i}} \cdot \sigma_{x_{i}^{\prime} y_{i^{\prime}}}} \text { pour } i \neq i^{\prime}
$$

En ce qui concerne l'écart-type des corrélations génétiques, nous avons utilisé la formule établie par ROBERTSON (I959):

$$
\mathrm{S}_{r_{g_{i} g^{\prime}}}=\frac{\mathrm{I}-v_{g_{i} g_{i}^{\prime}}^{2}}{2} \cdot \sqrt{\frac{\mathrm{S} h_{i} \cdot \mathrm{S} h_{i^{\prime}}}{h_{i}^{2} \cdot h_{i}^{2}}}
$$
$\left(r_{p} p_{i}^{\prime}\right)$

Les coefficients de corrélations phénotypiques entre les caractères mesurés chez les mères

$$
\begin{aligned}
& \gamma_{p_{i} p_{i^{\prime}}}=\frac{\sigma_{x_{i} x_{i}}}{\sigma_{x_{i}} \cdot \overline{x i}^{\prime}} \quad \text { pour } i \neq i^{\prime} \text { et chez les produits }\left(\gamma^{\prime} \boldsymbol{p}_{i} \boldsymbol{p}_{i}{ }^{\prime}\right) \\
& r^{\prime} p_{i} p_{i^{\prime}}=\frac{\sigma_{y_{i} y_{i} i^{\prime}}}{\sigma_{y_{i}} \cdot \sigma_{y^{\prime}}} \text { pour } i \neq i^{\prime}
\end{aligned}
$$

On peut par ailleurs calculer le coefficient de corrélation entre milieux :

$$
r_{e i e^{\prime}}=\frac{r_{p_{i} p_{i}^{\prime}}-h_{i} h_{i}{ }^{\prime} r_{g_{i t} g_{i}^{\prime}}}{e_{i} e_{i}{ }^{\prime}},
$$

pour $i \neq i^{\prime}$

en tenant compte du fait que :

$$
r_{p i p i^{\prime}}=h_{i} h_{i}^{\prime} r_{g_{i g} i^{\prime}}+e_{i} e_{i}{ }^{\prime} r_{e i e i^{\prime}},
$$

où $e_{i}$ et $e_{i}^{\prime}$ sont les coefficients de régression standardisés (" paths coefficients ") de WRIGHT (r92r et 1934) liés aux influences du milieu qui affectent ces caractères $i$ et $i^{\prime}$.

\section{III. — RÉSULTATS E'T DISCUSSION}

Nous présenterons et nous discuterons successivement :

- les critères de la variabilité phénotypique ou liée à l'influence du milieu,

- les paramètres génétiques proprement dits.

\section{I. - Variation phénotypique et liée au milieu}

3. II. Valeurs moyennes et écart-types.

Les valeurs moyennes des poids (tabl. 2) sont comparables pour la race charolaise à celles qui figurent dans d'autres études : Mc NALLY (I970) en Angleterre :signale, des poids à la naissance de $45 \mathrm{~kg}$ chez les mâles, de $40 \mathrm{~kg}$ chez les femelles (45 et 4I,6 respectivement dans notre étude). I,es poids au sevrage indiqués par cet auteur sont équivalents aux poids à 6 mois que nous enregistrons pour les mâles $(247 \mathrm{~kg})$ mais supérieurs pour les femelles $(229 \mathrm{~kg}$ contre $2 \mathrm{I} 4 \mathrm{~kg}$ ). De même, les résultats publiés par AURIOL et al. (I960) dans une étude préliminaire sur la race charolaise sont identiques pour le poids à la naissance : 45,8 $\mathrm{kg}$ dans le cas des 
veaux mâles et $4 \mathrm{I}, 8$ dans le cas des veaux femelles. Par contre, les poids à 6 mois indiqués par ces auteurs sont plus faibles $(223,6$ pour les veaux mâles et 207,3 pour les veaux femelles). Cette différence doit être vraisemblablement imputée en grande partie à 1'amélioration des conditions d'alimentation des veaux pendant la phase d'allaitement entre les années I958 et I968 qui représentent les dates moyennes de naissance des veaux respectivement dans cette étude et dans la nôtre.

\section{TABI,EAU 2}

Moyennes $(\mathrm{kg})$, écart-types $(\mathrm{kg})$ et coefficients de variation (p. Ioo) des caractères selon la vace et le sexe

\begin{tabular}{|c|c|c|c|c|c|c|c|c|c|c|}
\hline \multirow{2}{*}{ Race } & \multirow{2}{*}{$\begin{array}{l}\text { Indice du } \\
\text { caractere } \\
\text { (i) }\end{array}$} & \multicolumn{3}{|c|}{ Mères } & \multicolumn{3}{|c|}{ Descendants mâles } & \multicolumn{3}{|c|}{ Descendants femelles } \\
\hline & & $\overline{\mathrm{X}}_{\mathrm{i}}$ & $\sigma_{x_{i}}$ & $\mathrm{CV}$ & $\overline{\mathrm{Y}}_{i}$ & $\sigma_{y_{\mathfrak{i}}}$ & $\mathrm{CV}$ & $\overline{\mathrm{Y}}_{i}$ & $\sigma_{y_{i}}$ & $\mathrm{CV}$ \\
\hline \multirow{4}{*}{ 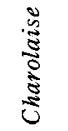 } & 1 & 42,3 & 4,8 & 11,4 & 45,0 & 5,1 & 11,3 & 41,6 & 4,5 & 10,9 \\
\hline & 2 & 135 & 15,8 & 11,7 & 144 & 17,3 & 12,0 & 130 & 14,1 & 10,8 \\
\hline & 3 & 165 & 18,8 & 11,4 & 178 & 21,7 & 12,2 & 159 & 17,1 & 10,8 \\
\hline & 4 & 222 & 24,0 & 10,8 & 247 & 30,8 & 12,5 & 214 & 22,6 & 10,6 \\
\hline \multirow{4}{*}{ 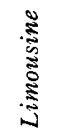 } & 1 & 37,5 & 3,8 & 10,2 & 38,6 & 3,3 & 8,6 & 36,0 & 3,4 & 9,4 \\
\hline & 2 & 121 & 12,4 & 10,2 & 125 & 13,6 & 10,8 & 115 & 11,9 & 10,3 \\
\hline & 3 & 148 & 14,7 & 9,9 & 155 & 16,5 & 10,6 & 141 & 14,3 & 10,1 \\
\hline & 4 & 201 & 19,1 & 9,5 & 219 & 22,8 & 10,4 & 192 & 18,4 & 9,6 \\
\hline
\end{tabular}

Références : Indices des caractères : 1, 2, 3 et $4=$ poids à la naissance, à 90 , à 120 et 180 jours, respectivement.

CV : Cœfficient de variation.

Les différences de poids moyens entre races charolaise et limousine : 6,4 et $5,6 \mathrm{~kg}$ à la naissance, I9,0 et $\mathrm{I}_{5,0} \mathrm{~kg}$ à 3 mois peuvent être comparées à celles enregistrées dans les expériences de croisement effectuées en France avec les taureaux de ces 2 races sur un même cheptel femelle, (VISSAC et al., I970). Ces différences varient entre 3,6 et $5,2 \mathrm{~kg}$ à la naissance, 9 et $15,5 \mathrm{~kg}$ à 75 jours. Elles sont par ailleurs de $4,9 \mathrm{~kg}$ à la naissance et de I2,I kg à 75 jours dans l'étude de PouJARDIEU et VISSAC (I968) consacrée à l'analyse de la variabilité des races charolaise et limousine en croisement. Ces valeurs sont dans 1'ensemble supérieures de près de $50 \mathrm{p}$. Ioo à la moitié des différences entre races pures que nous obtenons à la naissance et à 3 mois. Si on fait abstraction de l'influence des changements de condition d'élevage des femelles entre les races charolaise et limousine, changements dont l'incidence sur les poids à la naissance respectifs des veaux des 2 races doit être faible, trois explications peuvent être invoquées:

- une différence de valeur génétique entre les taureaux charolais utilisés par les centres d'insémination pour le croisement et ceux exploités en race pure par monte naturelle. Les premiers auraient un potentiel de croissance nettement supérieur aux seconds jusqu'à 3 mois. Ces 2 catégories présentent de fait, au premier abord, une différence de musculature très nette. Une telle fluctuation ne doit pas 
exister en race limousine où, comme nous l'avons précisé, la proportion de femelles soumise à 1'insémination est nettement plus élevée et où les mêmes types de taureaux sont en général utilisés par les centres en race pure et en croisement;

- une différence entre les effets non additifs qui se manifesteraient en croisement avec des taureaux charolais d'une part, limousins d'autre part, les premiers étant plus importants;

- un écart enfin dans les effets maternels des 2 races qui ne serait pas proportionnel aux différences de potentiel de croissance des veaux. On voit mal comment pourrait exister une grande discordance à cet égard dans le cas de races qui ne sont plus traites depuis longtemps et où les phénomènes de sélection naturelle ont dû assurer un équilibre harmonieux entre les besoins du jeune et les qualités d'élevage de sa mère (aptitude au vêlage, production laitière).

D'une façon générale, si les 2 dernières explications peuvent être retenues, en théorie, seule la première peut rendre compte, selon nous, d'un écart aussi important que celui enregistré quand on compare les veaux issus de taureaux charolais et limousins, en race pure d'une part, en croisement d'autre part.

On remarque, par ailleurs, que les poids des descendants femelles sont systématiquement inférieurs à ceux de leurs mères au même âge $(8$ et $9 \mathrm{~kg}$ à $\mathrm{I} 80$ jours en races charolaise et limousine respectivement). Cette différence pourrait être expliquée en invoquant un effet de l'âge : il s'agit vraisemblablement, d'un écart entre l'âge moyen des mères à la naissance de leurs produits et celui des grand mères maternelles lors de la naissance des mères de notre échantillon. Alors que l'âge moyen des grand mères devait être voisin de celui de l'ensemble de la population femelle, il n'en est pas de même pour celui des mères que nous avons retenues dans cet échantillon parce qu'elles avaient été contrôlées dans leur jeune âge : étant donné le développement récent des opérations de contrôle, on peut en effet penser que ces femelles étaient plus jeunes que la moyenne de la population. La différence d'effet maternel expliquerait donc les écarts de poids entre mères et produits femelles.

En ce qui concerne les différences de poids moyens entre sexes, $-3,4$ et $2,6 \mathrm{~kg}$ à la naissance, 33 et $27 \mathrm{~kg}$ à 6 mois, respectivement en charolais et limousin elles sont comparables entre elles en valeur relative ainsi qu'avec celles enregistrées sur races anglo-saxonnes pour le poids à la naissance (PETTY et CAR'TWRIGH'r, I966). Au sevrage, par contre, les mâles charolais et limousins ont un poids supérieur de I 5 p. IOo à celui des femelles contre 8 p. Ioo seulement dans le cas des races anglosaxonnes (tab1. 5). On pourrait expliquer cette différence en invoquant le traitement préférentiel auquel sont soumis les veaux mâles dans les troupeaux de sélection des races françaises ; ce phénomène est probablement moins accusé pour les races anglosaxonnes conduites de façon extensive et en grandes unités.

L'analyse des écart-types et coefficients de variation enregistrés est délicate car il peut exister une liaison générale moyenne - écart-type des poids entre sexes, entre races et entre les divers âges considérés. On sait que cette liaison exprime le fait que les processus de croissance sont partiellement liés à des effets multiplicatifs (Terssier, I955). L'étude de cette liaison permet néanmoins d'effectuer certaines observations :

- à poids moyens équivalents, l'écart-type des poids est supérieur en race charolaise; cela se traduit également par des coefficients de variation supérieurs pour cette race; 
H. A. MOLINUEVO, B. VISSAC

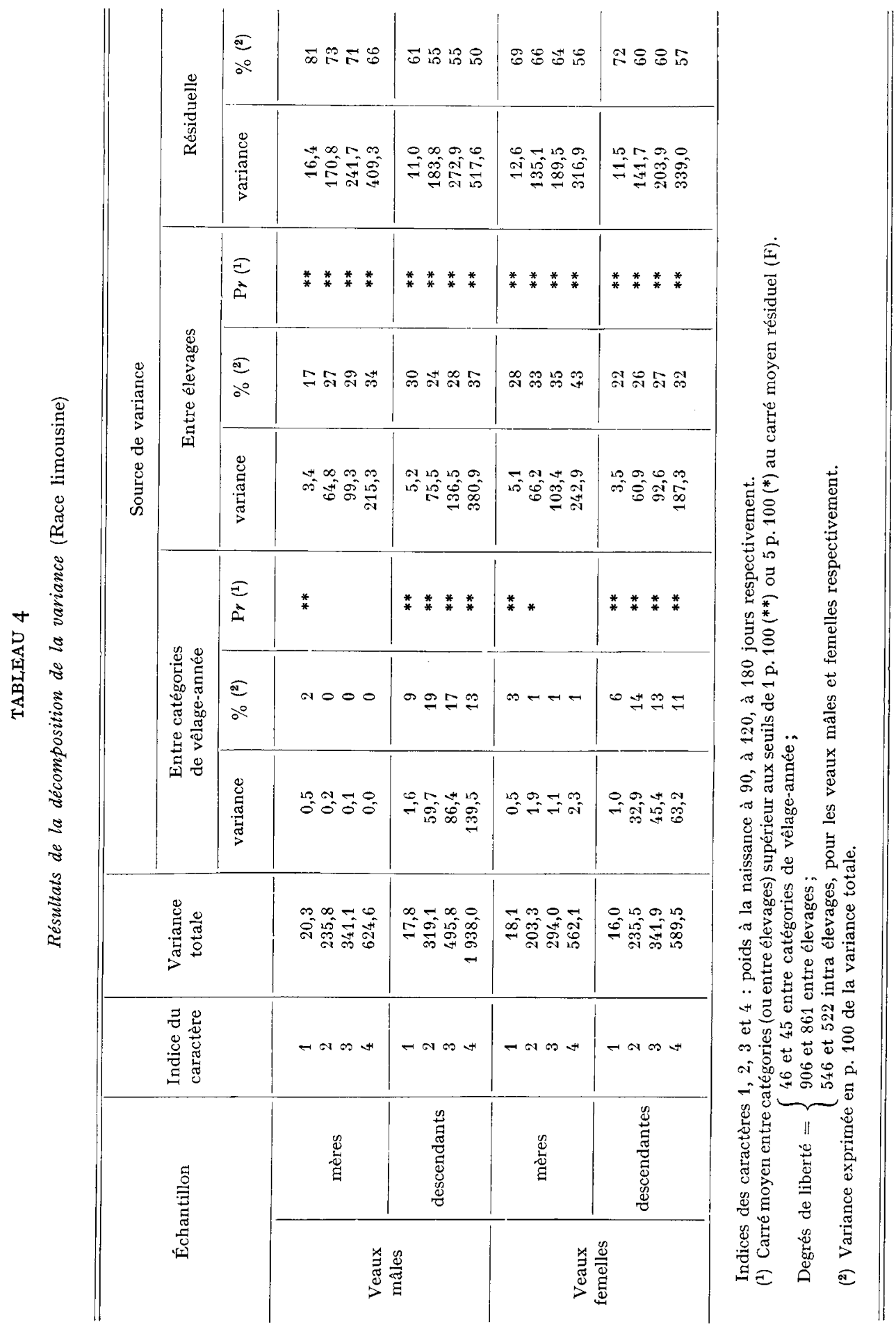


- quand on fixe également le poids moyen, les écart-types sont comparables pour les veaux mâles et femelles d'une race donnée, de la naissance à 3-4 mois ; à partir de cet âge, qui correspond au début du traitement préférentiel des veaux mâles, l'écart-type du poids de ces derniers augmente nettement plus vite que celui relatif aux veaux femelles. Quant au coefficient de variation, il croît pour les premiers de 4 à 6 mois mais demeure constant au contraire pour les seconds.

\section{TABLEAU 5}

Comparaison de l'effet du sexe sur les poids des veaux selon la race à viande

\begin{tabular}{|c|c|c|c|}
\hline \multirow{2}{*}{ Caractère } & \multirow{2}{*}{ Race } & \multicolumn{2}{|c|}{$\begin{array}{l}\text { Supériorité des mâles } \\
\text { par rapport aux femelles }\end{array}$} \\
\hline & & $\begin{array}{c}\text { Valeur additive } \\
\text { (kg) }\end{array}$ & $\begin{array}{c}\text { Valeur } \\
\text { multiplicative }\end{array}$ \\
\hline $\begin{array}{l}\text { Poids } \\
\text { à la naissance }\end{array}$ & $\begin{array}{l}\text { Anglo-Saxonne * } \\
\text { Limousine } \\
\text { Charolaise }\end{array}$ & $\begin{array}{l}2,1 \\
2,6 \\
3,4\end{array}$ & $\begin{array}{l}1,07 \\
1,07 \\
1,08\end{array}$ \\
\hline Poids au sevrage & $\begin{array}{l}\text { Anglo-Saxonne * } \\
\text { Limousine } \\
\text { Charolaise }\end{array}$ & $\begin{array}{l}17 \\
27 \\
33\end{array}$ & $\begin{array}{l}1,08 \\
1,14 \\
1,15\end{array}$ \\
\hline
\end{tabular}

* Races Aberdeen Angus, Hereford et Shorthorn (d'après PetTy et CARTWRIGHT, 1966).

\section{I2. Effets de l'âge, de l'année et de l'élevage sur les poids.}

Les différences entre catégories de vêlage qui regroupent les effets de l'âge de la mère et de l'année de naissance sont faibles en général et non significatives dans le cas des poids relatifs aux mères : la faible part de variance enregistrée dans ce cas (de I à 3 p. Ioo ) est due, comme nous l'avons signalé plus haut, au fait que les mères de même numéro de vêlage et vêlant la même année sont en général nées également la même année et dans le même élevage ; le renouvellement du cheptel est en effet assuré, la plupart du temps, intra-troupeau. Ce faible pourcentage fournirait donc une estimation par défaut des effets de l'année et du père sur la croissance des mères dans leur jeune âge. Dans le cas des descendants, les pourcentages de variance enregistrés sont évidemment plus élevés et très comparables d'un sexe à 1'autre, en race charolaise et en race limousine : de 6 à $9 \mathrm{p}$. Ioo sur le poids à la naissance, de I2 à I9 p. Ioo sur les poids à 3, 4 et 6 mois. Ces différences s'expliquent par le fait que, contrairement à ces derniers poids, le poids à la naissance n'est pratiquement pas influencé par l'année, tandis que l'effet de l'âge des mères, lié au milieu utérin ou au potentiel laitier se manifeste, lui, aussi bien à la naissance que sur les poids ultérieurs. On remarque enfin que la part de variance des poids entre catégories de vêlageannée reste constante de 3 mois à 6 mois en race charolaise; elle tend à diminuer au contraire en race limousine (de I9 à I3 p. Ioo chez les mâles, de I4 à II p. Ioo 
chez les femelles). Il est vraisemblable de penser que, dans cette dernière race, la conduite plus intensive et la complémentation plus précoce des animaux dès l'âge de 3-4 mois tendent à réduire les différences liées à l'année et à l'âge des mères.

Les fluctuations de l'effet de l'élevage intra-année et âge des mères sur la variance des poids sont comparables pour les veaux mâles et femelles, charolais et limousins : cet effet augmente avec l'âge ce qui est logique; les différences de traitement des animaux d'un troupeau à un autre ayant une répercussion d'autant plus grande sur la croissance que le veau n'est plus soumis à l'effet " tampon " du milieu maternel. On observe, en général, des différences entre élevages pour les poids à 3, 4 et 6 mois supérieures chez les mères des veaux limousins par rapport à celles relatives aux mères des veaux charolais, différences qui ne se retrouvent pas quand on compare les descendants de même sexe appartenant à chacune des 2 races. Ce résultat pourrait s'expliquer par le fait que les différences de conduite entre troupeaux, liées surtout en limousin à l'existence de troupeaux traditionnels et de troupeaux pratiquant l'élevage en plein air, tendent à se réduire avec le temps par la diminution progressive des premiers. Le système d'élevage des animaux limousins se rapproche ainsi de plus en plus du système d'élevage des charolais. On note enfin que la variance entre élevages des poids à $\mathrm{r} 20$ et I 80 jours tend à être plus élevée pour les veaux mâles que pour les veaux femelles : ceci traduit incontestablement les différences de complémentation des veaux d'une ferme à 1'autre, différences qui, nous l'avons précisé, concernent surtout les veaux mâles.

D'une façon générale, les variations entre élevages et catégories de vêlage observées dans notre étude sont comparables à celles enregistrées sous des climats et dans des pays très différents et qui sont présentées par Kumazaki et Matsuo (I967), Brown et Galvez (I969), Bosman et Harwin (I967), Cundiff et al. (I966), Warren et al. (I965), Niemann et Meydenrych (i965), Minyard et Dinkei, (I965), Vial, et MORE O'FARREL, (I965) entre autres.

\section{I3. Coefficients de corrélation.}

Pour ce qui est des corrélations phénotypiques (tab1. 6), on observe une tendance commune pour les 8 échantillons analysés (c'est-à-dire $=$ mères de descendants mâles ou femelles, descendants eux-mêmes pour chacune des deux races) ; les valeurs des coefficients diminuent graduellement, au fur et à mesure qu'augmente l'intervalle de temps entre les deux poids considérés. D'autre part, dans tous les échantillons étudiés, la valeur des corrélations est plus élevée entre les poids excluant le poids à la naissance : 0,8 à 0,9 contre 0,3 à 0,4 entre poids à la naissance et poids ultérieurs.

POUJARDiEu et VISSAC (I968) dans leur analyse du poids à la naissance, à 6o et 75 jours chez des produits croisés charolais et limousins, aboutissent à des résultats semblables aux nôtres. Dans la littérature, il existe, par ailleurs, une grande quantité d'information sur les corrélations phénotypiques entre le poids à la naissance et le poids au sevrage, en ce qui concerne les races britanniques. Nos résultats pour les races charolaise et limousine coïncident pleinement avec les résultats moyens que PETTY et CARTWRIGHT (I966) ont calculé d'après les travaux de Koch et ClARK (I955), LASLEY et al. (I96I), SWIGER (I96I), BRINKS et al. (I962), SHELBY) et al. (I963), PAhnish et al. (I964), BRInKs et al. (I964), FitzhuGH (I965), PETTTY I966). Ils sont, encore, équivalents à ceux mentionnés par KuMAZAKI et MAT- 
suo (rg67), entre le poids à la naissance et le poids au sevrage, chez les veaux d'une race japonaise. Nos résultats coïncident également de façon générale avec ceux que TAYLOR et CRAIG (I967) trouvent pour diverses mensurations corporelles prises chez des jumeaux de 3,6 et 9 mois ; ils sont par contre, légèrement supérieurs à ceux trouvés par SwIGER et al. (I962) dans leur expérience.

\section{TABLEAU 6}

Coefficients de corrélation phénotypique et due au milieu

\begin{tabular}{|c|c|c|c|c|c|}
\hline Race & $\begin{array}{l}\text { Indice du } \\
\text { caractère }\end{array}$ & 1 & -3 & $\begin{array}{l}\quad \\
3\end{array}$ & $3_{4}$ \\
\hline \multirow{4}{*}{ 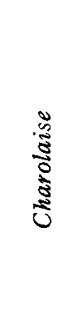 } & 1 & & $\begin{array}{l}0,388 \\
0,339\end{array}$ & $\begin{array}{l}0,341 \\
0,302\end{array}$ & $\begin{array}{l}0,309 \\
0,289\end{array}$ \\
\hline & 2 & $\begin{array}{l}0,381 \\
0,316\end{array}$ & & $\begin{array}{l}0,983 \\
0,982\end{array}$ & $\begin{array}{l}0,907 \\
0,895\end{array}$ \\
\hline & 3 & $\begin{array}{l}0,337 \\
0,261\end{array}$ & $\begin{array}{l}0,976 \\
0,972\end{array}$ & & $\begin{array}{l}0,950 \\
0,941\end{array}$ \\
\hline & 4 & $\begin{array}{l}0,286 \\
0,202\end{array}$ & $\begin{array}{l}0,874 \\
0,860\end{array}$ & $\begin{array}{l}0,935 \\
0,929\end{array}$ & \\
\hline \multirow{4}{*}{ 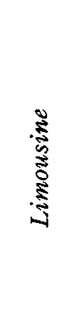 } & 1 & & $\begin{array}{l}0,381 \\
0,293\end{array}$ & $\begin{array}{l}0,345 \\
0,252\end{array}$ & $\begin{array}{l}0,306 \\
0,228\end{array}$ \\
\hline & 2 & $\begin{array}{c}0,388 \\
{ }^{(1)}\end{array}$ & & $\begin{array}{l}0,969 \\
0,961\end{array}$ & $\begin{array}{l}0,868 \\
0,835\end{array}$ \\
\hline & 3 & $\begin{array}{c}0,358 \\
\left({ }^{1}\right)\end{array}$ & $\begin{array}{c}0,974 \\
\text { (1) }\end{array}$ & & $\begin{array}{l}0,932 \\
0,899\end{array}$ \\
\hline & 4 & $\begin{array}{c}0,342 \\
\left({ }^{1}\right)\end{array}$ & $\begin{array}{c}0,846 \\
\left({ }^{1}\right)\end{array}$ & $\begin{array}{l}0,917 \\
0,286\end{array}$ & \\
\hline
\end{tabular}

Références : Les chiffres au-dessus et à droite des diagonales correspondent aux mâles; ceux à gauche et au-dessous des diagonales correspondent aux femelles. Dans chaque cellule, le chiffre supérieur est la corrélation phénotypique $\left(r_{p_{i}} \boldsymbol{p}_{i^{\prime}}\right)$ le chiffre inférieur est la corrélation due au milieu $\left(\boldsymbol{r}_{\boldsymbol{e}_{i} \boldsymbol{e}^{\prime}}\right)$.

(1) Le calcul n'a pas été réalisé car on obtenait des estimations de covariance négative sous le radical.

Nos résultats justifient en fait ce qu'on peut espérer sur le plan biologique, étant donné que les variations de poids à la naissance sont régies outre les effets des gènes du veau par des facteurs maternels différents de ceux agissant sur la croissance postnatale. En effet, ces derniers sont influencés par la capacité laitière de la mère qui les lie entre eux et qui n'affecte pas par contre le poids, à la naissance. De plus, il est logique de penser que ces poids, voisins dans le temps, soient plus exposés aux caractéristiques communes du milieu par rapport à d'autres enregistrés à des 
H. A. MOLINUEVO, B. VISSAC

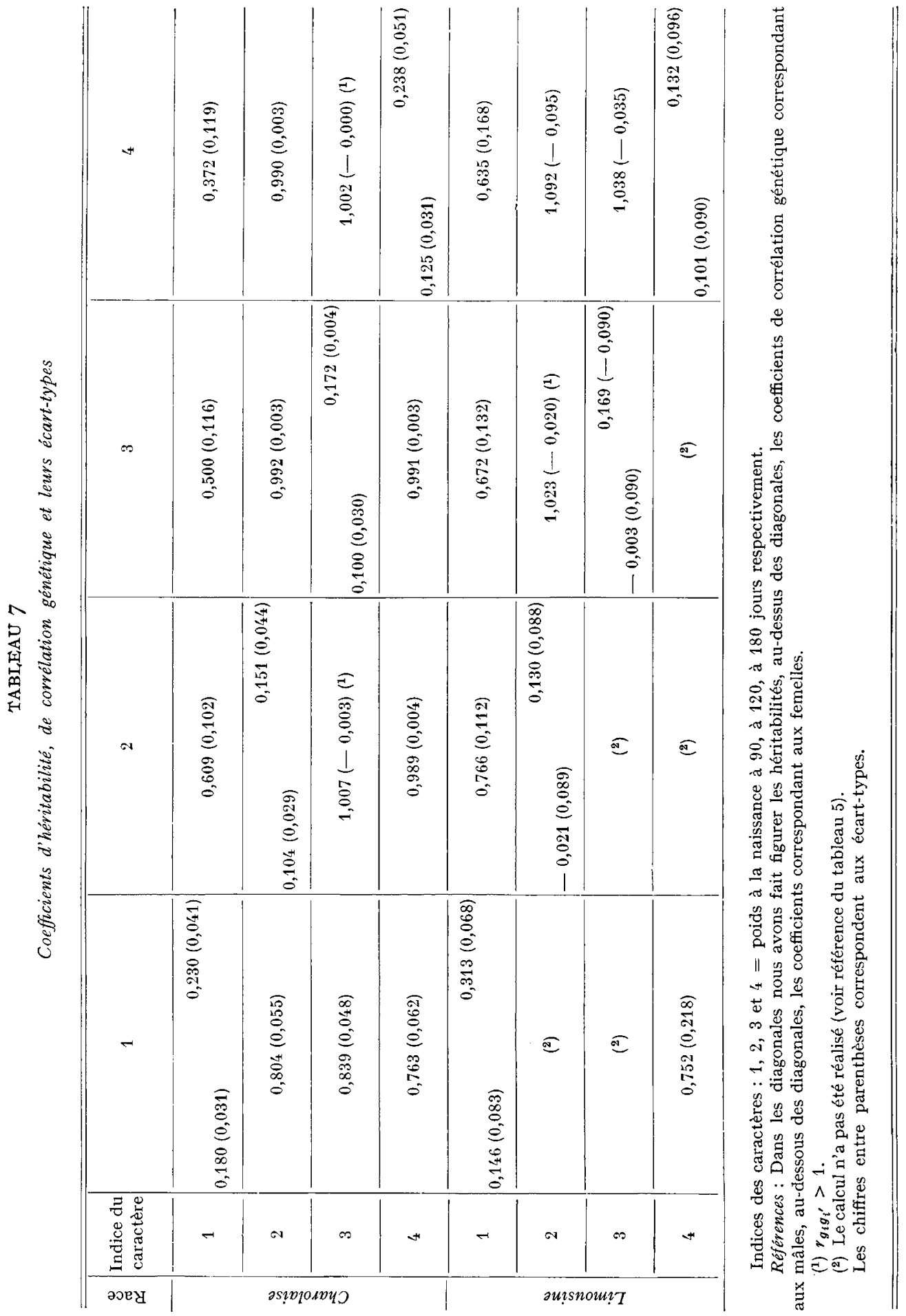


intervalles plus éloignés; pour cela, on peut s'attendre à trouver, comme c'est le cas, des corrélations phénotypiques plus élevées entre des poids rapprochés dans le temps qu'entre ceux plus distants. Mais on doit rappeler surtout que les poids après la naissance peuvent être liés arithmétiquement en ce qui concerne notamment les poids à 90 et I 20 jours obtenus souvent par interpolation linéaire à partir d'une pesée commune. Quant au poids à la naissance, fourni par l'éleveur lui-même, qui l'estime parfois subjectivement, sa variation est au contraire influencée par des erreurs spécifiques très importantes.

Ces observations sont confirmées d'une façon très nette par l'étude des corrélations dues au milieu figurant dans le tableau 6. Bien que ces corrélations soient de 1 'ordre de 0,2 à 0,3 entre le poids à la naissance et les autres poids enregistrés jusqu'au sevrage, celles obtenues entre les poids à 90 , I20 et I 80 jours varient entre 0,8 et 0,9 .

\section{2. - Paramètres génétiques}

\section{2I. Coefficients d'héritabilité.}

On envisagera successivement les variations des coefficients d'héritabilité du poids avec l'âge, le sexe et la race du veau.

D'après les résultats du tableau 7 schématisés sur la figure $r$, on remarque une tendance à l'obtention de valeurs d'héritabilité plus élevées pour le poids à la naissance que pour les autres poids. Le coefficient d'héritabilité du poids semble prendre vers 3-4 mois une valeur minimum et augmenter ensuite jusqu'au sevrage. On ne peut en fait conclure à l'existence de différences significatives entre les valeurs obtenues pour un même échantillon à des âges différents (tab1. 7).

PoujARDieu et Vissac (I968) notent également, sur des veaux croisés charolais et limousins, une tendance à la diminution de l'héritabilité du poids, pour la première race notamment, de la naissance à 3 mois. Quant aux estimations obtenues par les chercheurs américains sur races d'origine britannique et rassemblées par PETTY et CARTWRighT (I966), elles concernent les poids à la naissance et au sevrage, aucun travail portant sur un effectif élevé ne fournissant, à notre connaissance, des estimations d'héritabilité comparables pour les poids intermédiaires. Les valeurs d'héritabilité du poids à la naissance fournies par ces deux auteurs sont comprises en moyenne entre 0,40 et 0,44 ( 28 estimations) ; celles du poids au sevrage sont de 1'ordre de 0,3 (52 estimations). Plusieurs chercheurs : GrEGORY et al. (I950), ShELBY et al. (I957), Mahmud et Cobr (I963), Kumazaki et Matsuo (I967), Plasse et al. (I968), Kumazaki et Matsukawa (I963), Gregory (I957), obtiennent une héritabilité plus élevée pour le poids à la naissance que pour le poids au sevrage sur des échantillons d'animaux de différentes races et sous une grande diversité de climats. Par contre, pour KOCH et CLARK (I955 $a, b$ et $c$ ), LASLEY et al. (I96I), SNIGER et al. (I962), PAHNish et al. (I964), BRINKs et al. (I964), FiTzHuGH (I965), Swiger et al. (I965), PeitTy (I966), le coefficient d'héritabilité du poids est équivalent, voire légèrement plus élevé, au sevrage qu'à la naissance.

En fait, la tendance observée dans l'évolution des coefficients d'héritabilité du poids avec l'âge (fig. I) paraît logique dans la mesure où c'est à 3-4 mois que le poids du veau dépend le plus de la production laitière de sa mère (PouJARdiEu, I969). Cette diminution de l'héritabilité du poids de la naissance à 3 mois est peut- 
être accrue, d'ailleurs, par l'existence d'un coefficient de corrélation génétique négatif entre le potentiel de croissance du veau et la production laitière de la mère (RAY et al., I970) ; HOHENBOKEN et BRINKS, I97I). Le sevrage qui intervient progressivement à partir de 3-4 mois permet au veau d'ajuster ses propres besoins grâce au pâturage ou aux apports de concentré ; cela peut expliquer l'accroissement du coefficient d'héritabilité observé de 4 à 6 mois dans 3 cas sur 4 .

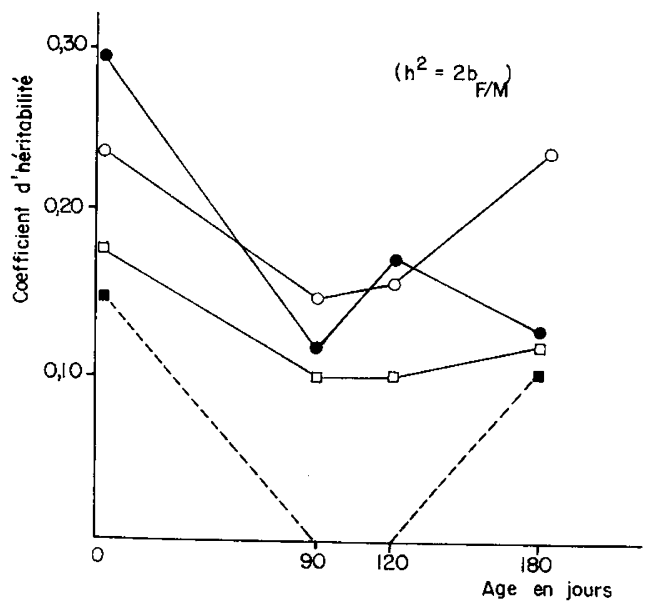

Fig x. - Évolution de l'héritabilité du poids, de la naissance au sevrage $\left(\hbar^{2}=2 b \mathrm{~F} / \mathrm{m}\right)$

$$
\begin{aligned}
& 0=\text { mâles charolais } \\
& \square=\text { femelles charolaises } \\
& -=\text { mâles limousins } \\
& \square=\text { femelles limousines }
\end{aligned}
$$

A l'examen de la figure I il semblerait, pour les femelles notamment, que les coefficients d'héritabilité du poids sont en général plus élevés en race charolaise qu'en race limousine, sauf toutefois pour le poids à la naissance. De la naissance à 3 mois, phase qui avait été considérée par Poujardieu et VISSAC (I968) pour estimer la variabilité génétique du poids entre les descendances croisées issues de taureaux charolais et limousins, la diminution d'héritabilité du poids paraît plus élevée dans le cas de la $2^{\mathrm{e}}$ race ; c'était au contraire la tendance inverse qui avait été signalée par les deux auteurs précédents. Une explication peut être avancée pour interpréter cette contradiction apparente. La production laitière souvent insuffisante des femelles limousines (PETrT, I97I) limiterait peut-être, plus fréquemment qu'en race charolaise, l'expression du potentiel de croissance des veaux. Ce ne serait pas le cas, en croisement, où on peut considérer que le potentiel laitier des. femelles de races mixtes accouplées au taureau limousin est en moyenne suffisant pour équilibrer les besoins du veau croisé, alors qu'il ne l'est souvent pas pour le croisé charolais issu de mères aux aptitudes comparables d'une part, et dont le potentiel de croissance est nettement plus élevé d'autre part.

Quant aux différences de valeur des coefficients d'héritabilité qui semblent apparaître entre les 2 sexes, les veaux mâles fournissant des coefficients plus élevés que les veaux femelles, elles peuvent recevoir 2 interprétations très différentes, l'une biologique, l'autre liée aux méthodes de calcul utilisées :

a) Si l'on admet l'existence de gènes influençant la croissance situés sur le chro- 
mosome X (ce qui a été suggéré par STONAKER (I963), dans une étude sur 1'hétérosis), il y aurait une corrélation génétique supérieure entre mère et fils qu'entre mère et fille. Ceci conduirait donc à une héritabilité plus grande pour les mâles, dans le cas où l'héritabilité est calculée à partir de la régression mère-produit.

Malheureusement, le fait de ne pas pouvoir inclure dans notre modèle l'effet des pères en partie confondu avec ceux de l'année, de l'élevage et de l'âge des mères nous a empêchés de calculer l'héritabilité par l'étude des demi-frères paternels, ce qui aurait permis de vérifier cette explication. Toutefois, ces différences d'héritabilité entre sexes sont notées également, mais elles sont moins nettes, dans l'étude de POUJARDIEU et VisSAC (I968) concernant la comparaison de demi-frères ou sœurs paternels. Thomas (I968) rapporte aussi des valeurs d'héritabilité plus élevées pour le poids au sevrage chez les mâles, dans une étude des liaisons entre les performances de demi-frères paternels. Vianna et al. (I964), de leur côté, effectuent une analyse de la variabilité du poids à la naissance dans un troupeau charolais et constatent la même tendance remarquée sur notre échantillon. En définitive, le fait qu'il n'existe pas, pour un même échantillon de races bovines à viande, de calculs d'héritabilité effectués simultanément par régression mère-produit et par analyse de variance entre demi-frères (ou sœurs) paternels, ne permet pas de conclure sur la validité de l'explication que nous avons suggérée.

b) Dans le modèle d'estimation de l'héritabilité, on utilise au dénominateur la variance phénotypique des veaux femelles (mères). Si, comme on peut le penser d'après les résultats ci-dessus, il existe une liaison entre la valeur moyenne du caractère et sa variabilité, celle-ci serait plus grande dans le sexe mâle, ce qui conduirait à une héritabilité plus élevée.

Nous avons réalisé un deuxième type de calcul de l'héritabilité en utilisant comme dénominateur la variance phénotypique des descendants et non des mères, et comme numérateur l'estimation de la variance génotypique par l'intermédiaire de la décomposition de la covariance :

$$
h_{i}^{2}=\frac{2 \sigma_{x_{i} y_{i}}}{\sigma_{y_{i}}^{2}}=\frac{\sigma_{g_{i}}^{2}}{\sigma_{y}^{2}}
$$

On élimine ainsi l'effet éventuel du sexe sur la variance des poids. Les valeurs ainsi obtenues sont exposées dans le tableau 8 , leur variation est schématisée sur la figure 2 ; elles diffèrent légèrement de celles antérieurement trouvées à 1'aide de la régression produit-mère, sans toutefois s'en éloigner d'une façon remarquable. Il semblerait néanmoins, au vu de ces résultats, et si l'on fait exception du poids à la naissance, que la $2^{\mathrm{e}}$ explication ci-dessus soit à retenir pour les écarts de valeurs d'héritabilité dans le cas des veaux mâles et femelles.

D'une façon générale, toutes ces différences de valeurs d'héritabilité du poids suivant 1'âge, le sexe ou la race dépendent des différences de variances phénotypiques résiduelles et génétiques qui apparaissent, pour un caractère donné, entre l'échantillon de mères et celui des produits, entre les descendants mâles et femelles, entre les veaux charolais et limousins. Comme nous l'avons observé pour la variance totale, les variances résiduelles augmentent régulièrement avec les moyennes, d'un échantillon à un autre (tabl. 3 et 4 ) entre sexes et entre races notamment. Les coefficients de variation (rapport de l'écart-type résiduel à la moyenne), plus stables, sont toujours plus élevés chez les mâles que chez les femelles et chez les charolais que 
chez les limousins. Ils suivent, par contre, des évolutions variées avec l'âge : en race limousine, ils passent par un maximum à 3 mois chez les mâles et les femelles; en charolais, ils augmentent régulièrement chez les mâles et diminuent chez les femelles.

\section{TABLEAU 8}

Coefficients d'héritabilité calculés par la formule

$$
h^{2}=\frac{2 \sigma_{x i y_{i}}}{\sigma_{y_{i}^{2}}^{2}}
$$

\begin{tabular}{|c|c|c|c|c|c|}
\hline \multirow{2}{*}{\multicolumn{2}{|c|}{$\frac{\text { Race }}{\text { Sexe }}$}} & \multicolumn{2}{|c|}{ Charolaise } & \multicolumn{2}{|c|}{ Limousine } \\
\hline & & Mâle & Femelle & Mâle & Femelle \\
\hline 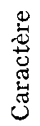 & $\begin{array}{l}1 \\
2 \\
3 \\
4\end{array}$ & $\begin{array}{l}0,218 \\
0,125 \\
0,130 \\
0,147\end{array}$ & $\begin{array}{l}0,199 \\
0,131 \\
0,120 \\
0,139\end{array}$ & $\begin{array}{l}0,468 \\
0,121 \\
0,149 \\
0,105\end{array}$ & $\begin{array}{r}0,160 \\
-0,020 \\
-0,002 \\
0,095\end{array}$ \\
\hline
\end{tabular}

Indices des caractères : $1,2,3$ et $4=$ poids à la naissance, à 90 , à 120 , à 180 jours respectivement.

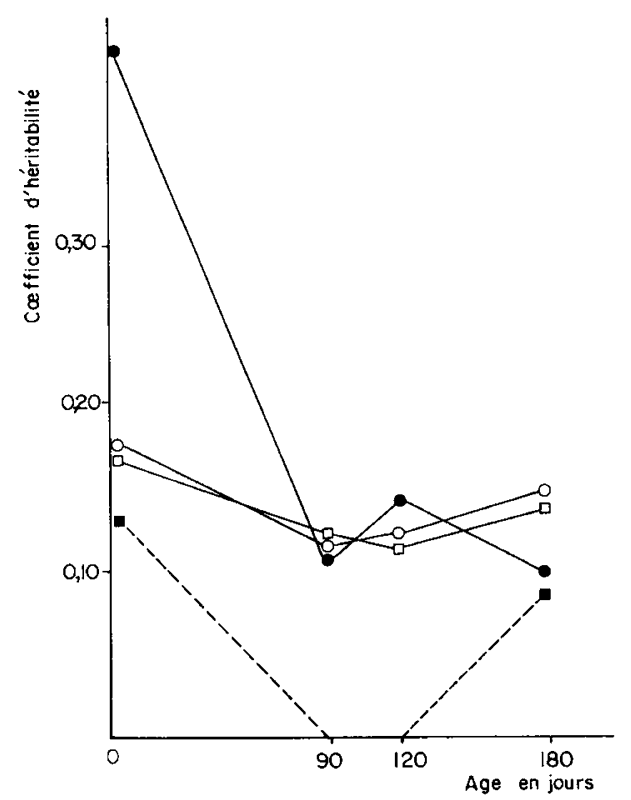

l'IG. 2. - Évolution de l'héritabilité du poids, de la naissance au sevrage $\left(h^{2}=\widehat{\sigma}_{g}^{2} / \widehat{\sigma}^{y}\right)$.

$0=$ mâles charolais

$\square=$ femelles charolaises

- = mâles limousins

- = femelles limousines 
En ce qui concerne les coefficients de variation génétique des poids (écart-type génétique/moyenne) $\times$ Ioo on observe les phénomènes suivants (tabl. 9) :

- valeurs inférieures en race limousine par rapport à la charolaise (le poids à la naissance des mâles mis à part) et pour les échantillons de femelles par rapport à ceux de mâles ;

- diminution systématique de la naissance à 3-4 mois avec remontée au-delà de 4 mois pour 2 échantillons.

La variation de l'héritabilité du poids avec l'âge s'explique donc par une double évolution en sens contraire des 2 composantes de ce coefficient : augmentation de o à 3-4 mois de la variance phénotypique, puis diminution ensuite, et inversement pour la variance génétique.

\section{TABLEAU 9}

Comparaison des critères de variabilité génétique du poids des veaux charolais et limousins

\begin{tabular}{|c|c|c|c|c|c|c|c|}
\hline \multicolumn{6}{|c|}{ Coefficient de variation génétique $\left({ }^{1}\right)$} & \multirow{2}{*}{\multicolumn{2}{|c|}{$\begin{array}{c}\text { Rapport } \\
\text { des variances } \\
\text { génétiques }\left({ }^{2}\right)\end{array}$}} \\
\hline \multirow{2}{*}{\multicolumn{2}{|c|}{$\frac{\text { Race }}{\text { Sexe }}$}} & \multicolumn{2}{|c|}{ Charolaise } & \multicolumn{2}{|c|}{ Limousine } & & \\
\hline & & Mâle & Femelle & Mâle & Femelle & Mâle & Femelle \\
\hline$\stackrel{0}{\leftrightarrows}$ & 1 & 5,3 & 4,9 & 5,9 & 2,9 & 1,1 & 2,2 \\
\hline$\stackrel{ \pm}{ \pm}$ & 2 & 4,2 & 3,9 & 3,8 & - & 1,7 & - \\
\hline 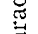 & 3 & 4,4 & 3,7 & 4,1 & - & 1,5 & - \\
\hline ن & $t_{t}$ & 4,8 & 3,9 & 3,4 & 3,8 & 2,6 & 2,2 \\
\hline
\end{tabular}

Indices des caractères : $1,2,3$ et $4=$ poids à la naissance, à 90 , à 120 , à 180 jours respectivement.
(1) $\frac{\mathrm{S}_{g_{i}}}{\bar{y}_{i}} \times 100$.
(2) $\frac{\mathrm{S}_{g_{i}}^{2} \text { (charolais) }}{\mathrm{S}_{g_{\imath}}^{2}}$ (limousin).

3. 22. Coefficients de corrélation génétique.

Ces coefficients sont nettement plus élevés que ceux relatifs aux corrélations phénotypiques et dues au milieu. Ils indiquent que 20 à 60 p. Ioo de la variance génétique des poids à 3,4 et 6 mois est liée à celle du poids à la naissance. Cette liaison est plus élevée en race limousine que dans le cas de la race charolaise notamment pour les veaux mâles. Ces 3 derniers poids, par contre, présentent entre eux des coefficients de corrélation génétique voisins de l'unité.

Les corrélations génétiques entre les poids à la naissance et au sevrage des races que nous avons analysées ne semblent pas différentes de celles signalées pour les races britanniques, d'après les travaux de Koch et CLARK (I955 a) LASLEY et al. (I96I) SwIGer (I96I) Brinks et al. (I962) Shelby et al. (I963) Pahnish et al. (I964) Brinks et al. (I964) Fitzhugh (I965), PetTty (I966). Ces résultats concordent aussi avec ceux trouvés par Kumazaki et Matsuo (I967) pour des races japonaises. Il convient de remarquer cependant que tous ces auteurs n'ont étudié que la liaison génétique entre des poids à la naissance et au sevrage. 


\section{CONCLUSION}

Les valeurs des paramètres génétiques obtenues dans cette étude sont, en définitive, comparables à celles enregistrées sur races anglo-saxonnes pour les poids à la naissance et au sevrage. L'évolution des coefficients d'héritabilité pendant la phase d'allaitement montre, en outre, l'intérêt qu'il y a à utiliser les poids à 3-4 mois pour estimer l'effet maternel, les poids à 6 mois pour estimer au contraire le potentiel de croissance des veaux.

Plusieurs faits indiquent que la variabilité génétique du poids, dans les échantillons étudiés, est plus élevée en race charolaise qu'en race limousine, et ce, tant en valeur absolue qu'en valeur relative. Quant à la possibilité d'améliorer dans ces 2 races la croissance post-natale indépendamment du poids à la naissance, Foulley et MolinUEvo (I97I) ont pu montrer, en considérant la sélection massale des veaux mâles sur le poids au sevrage, que les perspectives étaient plus favorables en race charolaise qu'en race limousine pour laquelle, il est vrai, la fréquence des accidents de parturition est actuellement plus réduite. Ce résultat découle logiquement des valeurs respectives des paramètres génétiques relatifs aux poids à la naissance et à 6 mois obtenus dans cette étude pour chacune des 2 races (érhantillons de veaux mâles) : $\left(\frac{h_{4}^{2}}{h_{1}^{2}}\right)$

- rapport des coefficients d'héritabilité des poids à 6 mois et à la naissance supérieur avec les mâles charolais $(\mathrm{I}, \mathrm{OO})$ qu'avec les limousins $(0,42)$.

- coefficient de corrélation génétique entre ces deux poids plus élevé avec les mâles limousins $(0,64)$ qu'avec les charolais $(0,37)$.

Reçu pour publication en mars 1972.

\section{REMERCIEMENTS}

Nous tenons à remercier les éleveurs et organismes d'élevage qui ont permis la collecte périodique des pesées de leurs veaux dans des conditions parfois très astreignantes, le personnel du centre de traitement de l'information du C. N. R. Z., B. Poujardieu pour l'aide qu'il nous a apportée dans l'élaboration et l'analyse statistique de cette information.

La lecture critique du manuscrit a par ailleurs été effectuée par B. BiBÉ et G. E. JOANDET auxquels nous exprimons notre gratitude.

\section{SUMMARY}

\section{GENETIC VARIABILITY OF PRE-WEANING GROWTH IN CHAROLAIS AND LIMOUSIN CATTLE}

I2 361 Charolais and 2928 Limousin dam-progeny pairs were constituted from the growth recording of beef calves. We had data on the weight of these pairs at birth, 90, I20 and I 80 days.

Heritability and genetic correlation coefficients between these criteria were estimated from dam-progeny regression coefficients within herd, year, and dam age group.

Heritability coefficients of weight which are usually highest at birth (0.15-0.30), go to $a$ minimum at about 3.4 months (0.00-0.17), and increase from 4 to 6 months (0.10-0.24). 
In general, these coefficients are higher for male calves than for femalescalves. This difference, which could be interpreted as due to sexlinked genes, was found to be mostly the result of deviation between sexes in phenotypic variance.

These coefficients, as well as genetic variability, expressed in absolute or relative value (coefficient of genetic variability) are usually higher in the Charolais breed for homologous weights. The values of genetic correlation are generally higher in the Limousin breed.

On the whole, these results agree with those obtained in other breeds, particularly british and american breeds. Their analysis allow us to anticipate that it will be easier to select Charolais male calves for post-natal growth independently of birth weight, than Limousin ones.

\section{RÉFÉRENCES BIBLIOGRAPHIQUES}

Auriol P., Dumont B. L., Duplan J. M., 1960. Caractéristiques générales des vaches charolaises et croissance de leurs produits. France-Elevage charolais, $28 \mathrm{p}$.

Belic M., Menissier F., I968. Étude de quelques facteurs influençant les difficultés de vêlage en croisement industriel. Ann. Zootech., 17, 107-142.

Bosman D. J., HaRwin G. O., 1967. Variation between herds in respect of the influence of year, sex, season and age of cow on weaning of beef calves. Proc. S. Afr. Soc. Anim. Prod., 6, 213-2I7.

Brinks J. S., Clark, R. T., Kieffer N. M., Quesenberry J. R., r962. Genetic and environmental factors affecting performance traits of Hereford bulls. J. Anim. Sci., 21, 777-78o.

Brinks J. S., Clark R. T., KIEFfer N. M., URICK J. J., r964. Estimates of genetic, environmental and phenotypic parameters in range Hereford females. J. Anim. Sci., 13, 7I I-7 6.

Brown C. J., Galvez M., 1969. Maternal and other effects on birthweight of beef calves. J. Anim. Sci., 28, 162-167.

Cundiff L. V., Willham R. L., Pratt C. A., I966. Factors to take into account when adjusting weaning weights of calves. Mis. Publs. Okla. Exp. Stn., No M. P. 78, 5-14.

Fitzhugh H. A. Jr., I965. A biometrical evaluation of weight of beef cows and performance of their progeny. Ph. D. Dissertation. Texas A. and M. University, College Station, Texas.

Foulley J. L., Molinuevo H. A., r97r. Possibilité de modification par sélection de la forme de la courbe de croissance avant sevrage des veaux de races à viande françaises. Communic. Journ. Féd. eur. Zootech., Paris-Versailles, $6 \mathrm{p}$. ronéoté.

Gregory K. E., I96r. Improvement of beef cattle through breeding methods. Res. Bull., I96, University of Nebraska, College of agriculture.

Gregory K. E., Blun C. T., Baker M. L., I950. A study of some of the factors influencing the birth and weaning weights of beef calves. J. Anim. Sci., 9, 338-346.

Hazel L. N., r943. The genetic basis for constructing selection indexes. Genetics, 28, 476-490.

Hohenвoken W. D., Brinks J. S., 197I. Relationships between direct and maternal effects on growth in Herefords : II. Partitioning of covariance between relatives. J. Anim. Sci., 32, 26-34.

Kосн R. M., CLARK R. T., I955a. Genetic and environmental relationships among economic characters in beef cattle. I. Correlation among paternal and maternal half-sibs. J. Anim. Sci., 14, 775-785.

Koch R. M., CLARK R. T., I955 b. Genetic and environmental relationships among economic characters in beef cattle. II. Correlations between offspring and dam and offspring and sire. J. Anim. Sci., 14, 786-79x.

Koch R. M., CLARK R. T., I955 c. Genetic and environmental relationships among economic characters in beef cattle. III. Evaluating maternal environment. J. Anim. Sci., 14, 979-996.

Kumasaki K., Matsukawa T., I963. Statistical and genetic studies on meat productivity of the Japanese breed of cattle. II. Estimates of the genetic parameters for body measurements and birth, weaning and 12 months of age. Bull. Chugoku. agric. Exp. Sta., B, n ${ }^{\circ}$ I I, 27-42.

Kumazaki K., Matsuo T., 1967. Genetic and environmental factors affecting birth weight and weaning weight of beef calves. A gric. Bull. Saga Univ., n ${ }^{\circ} 24,6 \mathrm{I}-7 \mathrm{I}$ (in Anim. Breed. Abstr., 37, 424-425).

Lasley F. F., Day B. N., Comfort J. E., I96x. Some genetic aspects of gestation length, and birth and weaning weights in Hercford cattle. J. Anim. Sci., 20, 737-74I.

Lush J. L., I940. Intra-sire correlations or regressions of offspring on dam as a method of estimation heritability of characteristics. Proc. Amer. Soc. Animal Prod., 23, 293-301.

Манмud А., Совв E. Н., 1963. Factors affecting weaning weights preweaning gains, and conformation scores of beef calves in Hawaii. J. Anim. Sci., 22, 820 (abstr.).

Mc Nally D. H., r97o. A note on the weights and weight, gains of some charolais and contemporary calves of other breeds in Northerm Ireland. Animal Prod., 12, 539-542.

Minyard J. Z., Dinkel C. A., I $965 a$. Weaning weight of beef calves as affected by age and sex of calf and age of dam. J. Anim. Sci., 24, ro67-1071. 
Minyard J. A., Dinkel C. A., I965 $b$. Heritability and repeatability of weaning weight in beef cattle. J. Anim. Sci., 24, I072-1074.

Niemann P. J., Heydenrych H. J., i965. Some environmental and physiological factors affecting birth and weaning weights of beef calves. Tech. Commun. Dep. Agric. tech. serv., Répub. S.- Afr., no $4 \mathrm{r}$, II p.

Pahnish D. F., Roberson R. L., Taylor R. L., Brinks J. S., Clark R. T., Roubicek C. B., ig64. Genetic analyses of economic traits measured in range-raised Herefords at preweaning and weaning ages. J. Anim. Sci., 23, 562-568.

Petit M., I971. Variations de la production laitière de vaches salers et limousines allaitant leurs veaux. Communic. Journ. Féd. eur. Zootech., Paris-Versailles, ro p.

Petty R. R. Jr., I966. A biometrical evaluation of growth and conformation traits and comparison of selection procedures of young British breed beef cattle. M. S. Thesis, Texas A and M University, College Station, Texas.

Petty R. R. Jr., Cartwright T. C., I966. A summary of genetic and environmental statistics for growth and conformation traits of young beef cattle. Depart. Tech. Rep., n ${ }_{5}^{5}$, Texas A and M University.

Poujardieu B., 1969. Recherche d'une méthode d'estimation de la production laitière des femelles. ovines et bovines pendant la phase d'allaitement. Ann. Zootech., 18, 299-3I5.

Poujardieu B., Vissac B., I968. Étude biométrique de la valeur bouchère de veaux croisés charolais et limousins. Ann. Zootech., 17, I43-I58.

Ray D. E., Roubicek C. B., Pahnish O. F., Brinks J. S., I97o. Breeding merit of topcross parents for preweaning traits in Hereford cattle. J. Anim. Sci., 30, I6I-I 66.

RÉGIS R., I969. Les variations d'ouverture pelvienne et leur relation avec les conditions de vélage Mémoire de fin d'études E. N. S. A., Grignon.

RoBertson A., I959. The sampling variance of the genetic correlation coefficient. Biometrics, 15, 469-485.

Shelby C. E., Clark R. T., Quesenberry J. R., Woodward R. R., r957. Heritability of some economic traits in record of performance bulls. J. Anim. Sci., 16, ro19 (abstr.).

Shelby $\quad$ C. E., Harvey W. R., Clark R. T., Quesenberry J. R., Woodward R. R., I963. Estimates of phenotypic and genetic parameters in ten years of Miles City R. O. P. steer data. J. Anim. Sci., 22, 346-353.

Stonaker H. H., I963. A genetic hypothesis for sex-mating system interactions in growth of cattle and poultry. J. Anim. Sci., 22, 320-325.

SWIGER L. A., x $96 \mathrm{r}$. Genetic and environmental influences on gain of beef cattle during various periods of life. J. Anim. Sci., 20, I83-г 88 .

Swiger L. A., Koch R. M., Gregory K. E., Arthaud V. H., Rowden W. W., Ingalls J. E., I962. Evaluating pre-weaning growth of beef calves. J. Anim. Sci., 21, 78I-786.

Swiger L. A., Gregory K. E., Sumption J. J., Breidenstein B. C., Arthaud V. H., i965. Selection indexes for efficiency of beef production. J. Anim. Sci., 24, 418-424.

Taylor St. C. S., Craig J., 1967. Variation during growth of twin cattle. Anim. Prod., 9, 35-60.

Teissier G., I955. Allométrie de taille et variabilité chez Maia squinado. Arch. Zool. exper. gen., 92, $22 \mathrm{I}-264$.

Tномаs R. C., I968. The genetic and environmental aspects of maternal effects upon weaning weight of beef calves. Diss. Abstr. B, 29, 1286-B. (In Anim. breed. Abstr., 37, 23 I).

Vial V. E., More O'Ferrall G. J., I956. The effects of breed, season and age of dam on the birth weight of 'dairy and crossbred calves and their growth during the first seven days of age. Ir. J.agric. Res., 4, 223-230.

Vianna A. T., De Alba J., Paes G., Macokfe C., I964. Mode of inheritance of birth weight and gestation lenght in charolais cattle. Turrialba, 14, I20-127.

Vissac B., I970. L'utilisation du charolais pour la production de femelles d'élevages. Bull. tech. Dép. Génét. anim. (Inst. nation. Rech. agron. Fr.) no 9.

Warren E. P., Thrift F. A., Carmon J. L., I965. Factors influencing weaning weights of Georgia beef calves. J. Anim. Sci., 24, 853-854 (abstr.). 